\title{
Medical Records Documentation of HIV/AIDS Clinical Services at Primary Health Care (PHC) Facilities and Its Implications on Continuum of Care and Operational Research in South Africa
}

\author{
H. N. Fomundam ${ }^{1}$, A. R. Tesfay ${ }^{1}$, S. A. Mushipe ${ }^{1 *}$, H. T. Nyambi', A. K. Wutoh ${ }^{2}$ \\ ${ }^{1}$ Howard University Global Initiative South Africa, Howard University, Pretoria, Republic of South Africa \\ ${ }^{2}$ Howard University, Washington DC, USA \\ Email: *abrahamrezene@yahoo.com, *smushipe@yahoo.com
}

How to cite this paper: Fomundam, H.N., Tesfay, A.R., Mushipe, S.A., Nyambi, H.T. and Wutoh, A.K. (2021) Medical Records Documentation of HIV/AIDS Clinical Services at Primary Health Care (PHC) Facilities and Its Implications on Continuum of Care and Operational Research in South Africa. World Journal of AIDS, 11, 60-70. https://doi.org/10.4236/wja.2021.112006

Received: August 26, 2020

Accepted: June 15, 2021

Published: June 18, 2021

Copyright $\odot 2021$ by author(s) and Scientific Research Publishing Inc. This work is licensed under the Creative Commons Attribution International License (CC BY 4.0).

http://creativecommons.org/licenses/by/4.0/

\begin{abstract}
Background: Patients medical records are used to document care processes for communication amongst healthcare workers for continued patient management. Incomplete or inaccurate documentation can adversely affect the quality of patients' care, leading to medication and treatment errors, increased morbidity, and mortality. Quality documentation in medical records is therefore an essential component of optimal healthcare and facilitates an individual's continuity of care. This study aimed to assess the quality of documentation of clinical data through the review of the accuracy and completeness of clinical records among newly diagnosed HIV-positive persons. The study is a sub analysis of a prospective longitudinal study that followed a cohort of 12,413 persons who were newly diagnosed with HIV infection. Severe limitations in retrieving reliable information and data became an obstacle to our research and led the study team to conduct medical records documentation and data audit to verify the accuracy and completeness of the data for newly diagnosed HIV positive persons. Methods: A cross-sectional study was conducted using routine data generated from 75 randomly selected newly diagnosed HIV positive persons aged 12-years-old and above between June 1, 2014 and March 31, 2015 in 36 purposively selected primary health care (PHC) clinics in South Africa. The facilities were selected from three high HIV-burden districts of South Africa (Gert Sibande, uThukela and City of Johannesburg). Results: Significant differences in the accuracy and completeness of clinical records were observed between data generated through the self-assessment by the facility managers and data primarily collected through review of the patients' clinical stationery and facility registers. $80 \%$ of
\end{abstract}


the newly diagnosed HIV positive persons were not documented as screened for tuberculosis (TB) on the clinical chart and $69 \%$ of newly diagnosed clients were not clinically staged (WHO staging). Furthermore, $80 \%$ of newly diagnosed HIV positive persons' follow up visit dates were not documented in the patient's clinical chart. Completeness of the data elements on the case record forms ranged from as low as $26 \%$ to a maximum of $66 \%$. It was noteworthy that all the clients' information documented in HIV counselling and testing registers, continuum of care registers and clinical charts were only partially completed. Conclusion: Each of the health care facilities under study had some significant gaps in medical records documentation of clinical data on newly diagnosed HIV positive persons. Data and information accuracy and completeness were a serious challenge in most facilities during the period under investigation. Of interest was the inconsistency of data recorded in the HCT registers, continuum of care and clinical charts of individual patients. This is a major impediment to HIV/AIDS comprehensive care.

\section{Keywords}

Accuracy, Completeness, Continuum of Care, Data, Documentation, HIV

\section{Introduction}

A complete and well-documented patient medical record ensures that the patient receives the best available care, and that good quality information is shared among health care providers, prevents ambiguity, and improves communication [1]. Conversely, incomplete, or inaccurate medical record documentation can adversely affect the quality of patients' care, leading to medication and treatment errors, and serious injury or death of a patient [2]. Medical records are a portal through which the healthcare providers can access the treatment history of a patient to learn about previous care, understand current diagnosis and status, and subsequently inform the formulation of a treatment plan [3]. Although medical record documentation has been studied for a long time, this paper will focus on how accurately and completely the data is being captured in the selected health care facilities.

As part of the surveillance of newly diagnosed Human Immunodeficiency virus (HIV) positive persons in South Africa, Howard University (HU) conducted a data quality assessment in 36 selected primary health care (PHC) facilities. The HU study team implemented the assessment, by reviewing clinical stationery of newly diagnosed HIV positive persons. The clinical stationery/patient chart is a comprehensive medical record kept at all the healthcare facilities, which contains valuable information regarding the patient's medical condition and management. Other medical records that were reviewed during the study were HIV counselling and Testing (HCT) registers, continuum of care registers, tuberculosis (TB) registers, Isoniazid Preventive Therapy (IPT) registers, laboratory results (such as CD4 count), and cotrimoxazole prophylactic therapy (CPT) registers. 
A semi-structured questionnaire was initially developed to measure validity, reliability, timeliness, precision, and integrity of data, through gathering data based on the perceptions of the facilities' managers. However, the results from the self-assessment were inconsistent, which influenced $\mathrm{HU}$ to further conduct an accuracy and completeness of the data review across the clinical records and clinical stationery of newly diagnosed HIV positive persons.

\section{Background}

Good quality medical records are an important component of safe and effective healthcare delivery [3]. The main function of the medical record is to serve as a central repository for planning and documenting patient care. Medical records also serve as for a means of communication between the patient and the health care provider, as well as among the providers and other professionals involved in the patient's care [4]. In low- and middle-income countries (LMIC) where access to and utilization of HIV prevention, care and treatment services has greatly reduced HIV transmission, the success of antiretroviral therapy (ART) scale-up also hinges on adequate medical record documentation of the patient history and care [5]. However, data collection and utilization at the health facilities in Africa are poor [6]. At the primary health care levels, where all data are captured and generated, there is still inadequate capacity of health workers in terms of skills to record accurate data, conduct the analysis, and effectively use the information for decision-making [6]. Generally, the African public health care system is understaffed and carries a heavy workload for its health workforce [7]. Supportive supervision for districts by ministries of health $(\mathrm{MoH})$ and district health teams for primary health facilities is also inadequate due to poor funding, save for implementing partners that channel donor funds to the national health care system and improve data quality [7]. Studies have shown an increasing recognition of the need to improve documentation of continuum of care for people living with HIV [8], and that clinical documentation could be improved, on average, in more than $50 \%$ of medical charts [9] [10].

Documentation is regarded as an essential element in the health system; failure of which is considered a significant breach of and deviation from the standard of care [11]. Incomplete and inaccurate data hampers the provision of high-quality care for people living with HIV (PLHIV), their treatment monitoring over time and their retention in care [12].

If health care services provided to patients are not captured, patient care and data integrity suffer immensely [13]. It is essential, therefore, that the correct amount of detail be available in medical health records to facilitate continuity in HIV care and treatment. Data for PLHIV helps understand HIV/AIDS/TB concerns and supports decision making to improve public health [14]. All healthcare providers have the responsibility to ensure that records are accurate and complete to effectively manage PLHIV/TB [15]. However, there have been concerns that efforts to increase quality of documentation may lead to increased 
workload on the part of healthcare providers and this may affect the qualityof-service delivery [4].

Howard University conducted a review of medical records to verify the accuracy and completeness of the available data. Accuracy was defined as the ability to track, using selected clinical records, the newly diagnosed HIV positive clients from diagnosis (HCT register) through to continuum of care registers, clinical stationery, and laboratory registers. The review also verified the availability of up-to-date and consistent information in the registers on each patient by making sure the value of the indicator was consistent for the same date. On the other hand, HU defined completeness as the extent to which existing data sources could complete the case record forms (CRF).

\section{Methods}

Study Design: This data quality review was conducted as a cross-sectional study using data generated from newly diagnosed HIV-positive persons' clinical stationery/records and medical registers in the 36 selected primary healthcare facilities.

Study Setting: This study was conducted in 36 primary health care facilities purposefully selected from three high HIV-burden districts of South Africa (Gert Sibande, uThukela, and City of Johannesburg). The health care facilities were selected based on the prevalence of HIV, HIV positivity rate and quality of clinical data available in the clinics.

Study Population: This study focused on the quality of clinical data of newly

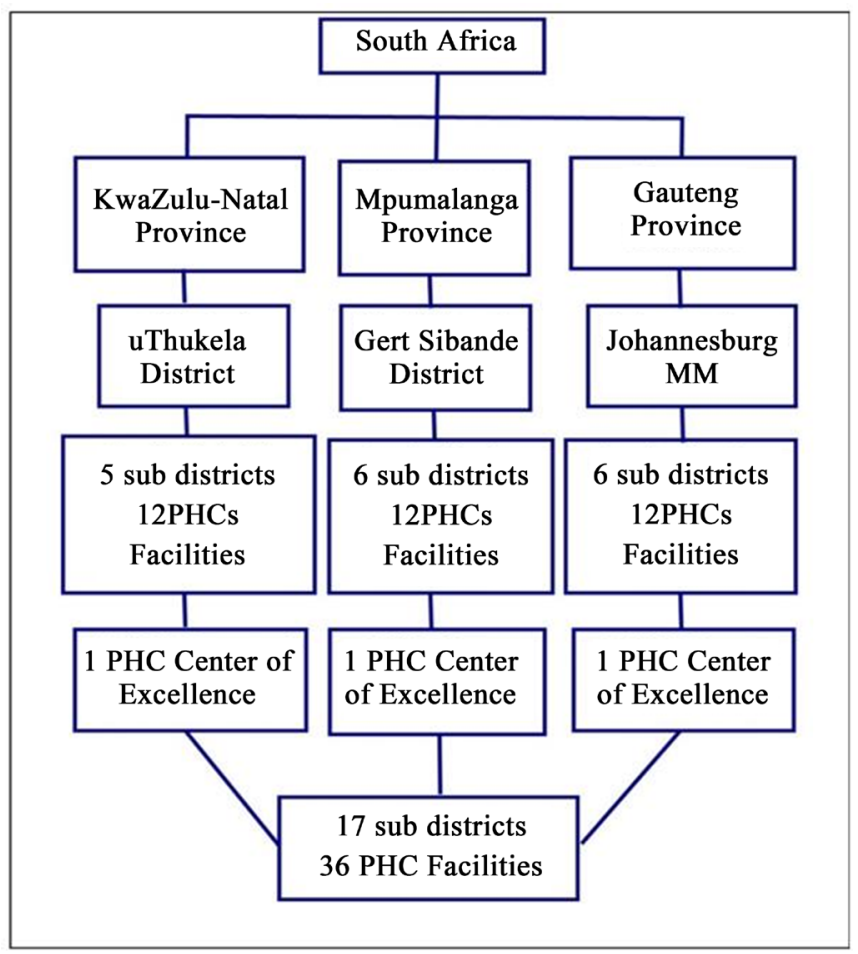

Figure 1. Study setting \& sampling framework. 
diagnosed HIV-positive persons. The study did not involve any direct interaction with clients who tested positive for HIV. Secondary data of newly diagnosed HIV positive persons 'clinical records were obtained from participating facilities. The study population for the assessment included newly diagnosed HIV positive persons aged 12 years and above. Individuals with an open clinical file were randomly selected in each facility. A convenience sample of 75 randomly selected individuals was drawn from 36 health facilities as indicated in Figure 1.

Data collection: This study sought to investigate the accuracy and completeness of clinical data of newly diagnosed HIV-positive persons. The initial solicitation of information was conducted through a semi structured questionnaire, which was developed to assess the quality of clinical data for newly diagnosed HIV positive persons and the capacity of selected facilities to retain patients in the continuum of HIV care. The gathering of the clinical data was based on the perceptions of the facility managers pertaining to newly diagnosed HIV positive persons.

Accuracy of data for newly diagnosed persons was assessed by comparing the consistency of specific data elements for individual patients across various records and registers such as the HCT registers, continuum of care registers, tuberculosis (TB) registers, isoniazid preventive therapy (IPT) registers, laboratory results such as $\mathrm{CD} 4$ count, cotrimoxazole preventive therapy (CPT) registers. The study case record form (CRF) was developed based on the national HIV management guideline in SA. The CRF contains the most important data elements for clinical care. The degree of completion of all the abstracted data from the patient clinical record onto the CRF determined the level of completeness. We completed 75 initial CRFs and 75 corresponding follow-up CRFs for randomly selected patients' clinical stationery/ records who tested HIV positive in January 2014.

Data analysis: We assessed completeness by computing the data elements across clinical care registers. Accuracy was assessed by computing consistency of data utilizing the standardised case record forms. Accuracy and completeness were presented in percentage values. Demographic variables were summarised in a table format.

Data Quality Management: Several measures were taken to minimize information bias and to ensure that quality data was collected. Data abstractors and data coordinators were trained on the importance of data quality, the use of the data collection tools (case record form), and the protection of participant privacy and confidentiality. The data collection tools were piloted using selected case record forms (CRF) that were not included in the study. The CRFs were validated at facility, district, and provincial levels. The data was then analysed manually after verification.

Ethical Considerations: Ethical approval was obtained from the ethics committee at the School of Health Systems and Public Health, at the University of Pretoria. Non-research determination was granted by CDC to conduct this DQA 
as an operational evaluation activity.

\section{Results}

The data quality assessment indicated that each of the 36 facilities had copies of the national standardised clinical stationery (Chart). The clinical chart was meant to be used to document services provided to HIV positive persons, who were eligible and enrolled to receive ART. Improvised tools were used to document services provided to HIV positive persons who were not enrolled on ART. All newly diagnosed HIV positive persons had their HIV test results recorded and documented in the HCT register. However, of interest, was the inconsistency of data recorded in the HCT, continuum of care and clinical chart of a given patient. Meaningful comparison was nearly impossible because none of these records were complete. Some patients were recorded in the HCT register but did not appear in the HIV continuum of care register and vice versa. Some patients had a clinical chart that was barely completed, and the only clinical record that was obtained was from the HCT register. In most instances, CD4 and TB data elements were only documented in the clinical chart; in some cases, the data elements documented in the clinical chart did not correspond with what was documented in the CD4 or TB registers.

Follow up visit dates were not documented in $80 \%(60 / 75)$ of the cases and in $100 \%(75 / 75)$ of the cases, clients' information documented in HCT register, pre-ART and clinical charts was partially complete. In some facilities, the clinical stationery was barely completed with significant missing data elements. Most facilities did not have adequate HIV care follow up data in their clinical records to complete the case record forms (CRFs). Completeness of the data elements of the CRF ranged from as low as $26 \%$ to a maximum of $66 \%$. Clients' laboratory results, such as $\mathrm{CD} 4$ count and TB were not available in the patients' folders in all 36 facilities. The use of improvised tools to document patients' information was employed in all the 36 facilities. Clinical data recorded in makeshift tools made it difficult to understand and interpret the data. Further, all 36/36 the facilities did not use the standardized registers and tools as prescribed by the district.

All (75/75) newly diagnosed HIV positive clients did not have documented CPT eligibility assessment services However, all (75/75) newly diagnosed HIV positive patients had documented IPT services in the IPT register. Table 1 below elaborates these results.

In some facilities, services for newly diagnosed HIV positive persons were not properly documented as a reflection of service provision, while in other facilities headcounts were tallied, but not properly documented in relevant registers. The results of the study also reflected $80 \%(63 / 75)$ of the newly diagnosed HIV positive clients who were not documented as screened for TB on the clinical chart and $69 \%(52 / 75)$ cases of newly diagnosed HIV positive clients who were not clinically staged (WHO staging). The clinical staff would verbally report that they tested a certain number of patients in each month but were unable to back 
Table 1. Summary of accuracy and completeness of data reviewed in the 36 primary health clinics.

\begin{tabular}{lc}
\hline Documentation of Services provided to Newly diagnosed HIV positive persons & Findings (\%) \\
\hline Availability of standardized patients' Clinical Chart & $36 / 36(100 \%)$ \\
Documented TB screening services per the national guidelines & $12 / 75(16 \%)$ \\
Documented clinical staging services (WHO staging) & $23 / 75(31 \%)$ \\
Documented follow up visit dates for newly diagnosed HIV positive persons & $15 / 75(20 \%)$ \\
Complete documentation of services in the HCT register \& patient clinical chart. & $0 / 75(0 \%)$ \\
Documented CD4 count results in the patients' clinical chart & $20 / 75(27 \%)$ \\
Documented CPT eligibility screening services in patients 'clinical chart. & $0 / 75(0 \%)$ \\
Documented IPT services in the IPT register & $75 / 75(100 \%)$ \\
Use of non-standardized tools to document patients' information & $36 / 36(100 \%)$ \\
\hline
\end{tabular}

up their statements with documented evidence.

\section{Discussion}

Our study findings indicated that all health care facilities under study had some significant gaps in medical record documentation of newly diagnosed HIV positive persons. Review of clinical charts demonstrated that most patients had missing data or clinical stationery that was partially documented. This omission became an obstacle to research; hence an audit was conducted to verify if data documented was accurate and complete. It was further observed that the health care workers were comfortable using improvised registers, hence noncompliance on the use of the standardised clinical stationery. A systematic review of published research results on non-compliance in filling medical records in hospitals throughout Indonesia showed that, non-compliance was mainly due to lack of competent human resources, no medical printing tool, non -availability of the medical documentation, and non-appeal to complete medical records [16]. It is therefore essential to strengthen the capacity of the ministry of health $(\mathrm{MoH})$ and the district health systems through capacity building, improving staffing, and regularly supporting supervision on data documentation [9].

A study also conducted in the United States of America reported that 98,000 patients died each year from medical errors [17]. Besides ethical and legal implications of non-documenting patients' information, this practice may also lead to loss to follow up [18], where return dates for the patient are not recorded with consequent late presentation for HIV care. The absence of data documentation of newly diagnosed HIV positive patients, who did not qualify to be initiated on ART, further led to missing laboratory results (CD4 count and TB results) and all (75/75) the newly diagnosed HIV positive clients were not documented as qualifying for $\mathrm{CPT}$ as required.

Each of the health care facilities involved in this study relied on TIER.Net which is a Department of Health (DOH) electronic patient management system 
(EPMS) used to capture data for PLHIV who have been enrolled on ART care. However, none of the facilities captured data on newly diagnosed HIV positive persons electronically during the period of the study. The EMR can be a good substitute for the paper-based medical records system [19], which equally requires adequate input of accurate data. However, as highlighted in other studies, it is essential to include the health care workers during the design and development of the software, so as to incorporate all the features that can promote a smooth transition from a paper-based system to an electronic system [19].

The findings of the study indicate that there was evidence of non-consistency of data recorded from the HCT registers to the clinical stationery, which led to a failure to make meaningful comparison for the documented data. The DQA showed a huge disparity between the services offered to PLHIV who were eligible for ART and patients requiring HIV continuum of care. There was no continuity of clinical data documentation from the HCT registers to the clinical stationery. Our results also confirmed other findings on non-consistency of data documentation, which led to a less accurate and incomplete assessments [20].

Findings from our study noted that health care personnel had to use nonstandard /improvised clinical records/documentation tools because of shortage of the official clinical stationery. These findings are consistent with similar studies which reported that medical personnel working in public hospitals experience record-keeping as a challenging activity owing to a shortage of recording material in resource limited settings [7]. This results in information missing from the patient files, and consequently incomplete data. Even with the use of EMR, health care workers may also be faced with challenges of power outages which lead to non-documentation in some cases.

The study further highlighted that all newly diagnosed HIV positive clients were not documented as qualifying for CPT, which is an essential component in prevention of opportunistic infections in HIV-infected persons [21]. Health care worker hesitancy has been established in several studies as one of the key issues associated with not effectively implementing CPT among HIV positive persons [21]. Findings in a study in Tanzania, where the implementation of cotrimoxazole by health care workers was exceptionally low, are in line with our study findings [22].

Standardisation of a data quality assessment allows for comparison of performance across health care facilities, which enables the formulation of benchmarks for appropriate care and identifying best practices in HIV care delivery. However, in this study, there is evidence that the clinical charts/ stationery available were not standardised. For PLHIV to fully benefit from ART, their clinical care needs to be documented along the continuum of care, patients need to be engaged in regular HIV care, and receive and adhere to effective antiretroviral therapy [10].

The need for comprehensive record-keeping remains fundamental in all healthcare facilities, to improve patient care. The present study confirms the findings 
that record-keeping in these settings was inadequate. It is recommended that there should be continuous training, monitoring and evaluation of health providers regarding record-keeping issues [20]. Supply of adequate recording materials and proper time management amongst nurses to improve record-keeping is essential and requires to be implemented in all health care facilities [23]

\section{Conclusion and Recommendations}

Promoting appropriate and sustainable data collection methods, databases, and systems to document all newly diagnosed HIV positive persons should be a priority in the context of scaling up ART. Patients could benefit both directly and indirectly from improved data quality, since accurate clinical data are a prerequisite for high standards of care, monitoring and consequently supporting patient retention in HIV care.

Health care providers should ensure that adequate documentation of all newly diagnosed HIV positive persons, and completeness of patient information should be a priority for all patients. Primary health care facilities should use patient information to guide patient care and improve outcomes. Standards must be applied to the data and the systems themselves to allow for and facilitate the exchange of data between various sources. It is also recommended that there should be continuous training, monitoring and evaluation of all the healthcare providers (nurses, data capturers) on record-keeping issues.

\section{Limitations}

The findings of this study should be considered in light of methodological limitations. The first limitation is that, as is the case with qualitative research, there is no necessary claim of generalizability of the findings to all sites caring for newly diagnosed HIV positive persons. Second, the gaps that were identified were also not necessarily prominent in all the facilities of all the districts. However, findings provide an indication of issues to consider for further strengthening of the health care system with regards to medical data documentation of persons living with HIV. The authors note additionally that there have been some clinical and data documentation improvement implemented since the study was conducted.

\section{Conflicts of Interest}

The authors declare no conflicts of interest regarding the publication of this paper.

\section{References}

[1] Rodziewicz, T.L., Houseman, B. and Hipskind, J.E. (2020) Medical Error Prevention. StatPearls Publishing, Treasure Island. [Internet]

[2] Campbell, P., Torrens, C., Pollock, A. and Maxwell, M. (2018) A Scoping Review of Evidence Relating to Communication Failures That Lead to Patient Harm. Glasglow Caledonia Univeristy, Glasglow; Univeristy of Stirling, Stirling. 
[3] Middleton, B., Bloomrosen, M., Dente, M.A., Hashmat, B., Koppel, R., Overhage, J.M., et al. (2013) Enhancing Patient Safety and Quality of Care by Improving the Usability of Electronic Health Record Systems: Recommendations from AMIA. Journal of the American Medical Informatics Association, 20, e2-e8. https://doi.org/10.1136/amiajnl-2012-001458

[4] Boonstra, A. and Broekhuis, M. (2010) Barriers to the Acceptance of Electronic Medical Records by Physicians from Systematic Review to Taxonomy and Interventions. BMC Health Services Research, 10, Article No. 231. https://doi.org/10.1186/1472-6963-10-231

[5] Venter, F. (2012) HIV Treatment in South Africa: The Challenges of an Increasingly Successful Antiretroviral Programme-Service Delivery. South African Health Review, 2012/2013.

[6] Fraser, H., Biondich, P., Moodle, D., Choi, S., Mamlin, B. and Szolovits, P. (2013) Implementing Electronic Medical Record Systems in Developing Countries. Innovation in Health Informatics, 13, 83-95. https://doi.org/10.14236/jhi.v13i2.585

[7] World Health Organization (2012) Management of Patient Information Trends and Challenges in Member States: Global Observatory for eHealth Series. World Health Organization, Switzerland.

[8] Kranzer, K., Govindasamy, D., Ford, N., Johnston, V. and Lawn, S.D. (2012) Quantifying and Addressing Losses along the Continuum of Care for People Living with HIV Infection in Sub-Saharan Africa: A Systematic Review. Journal of the International AIDS Society, 15, Article ID: 17383. https://doi.org/10.7448/IAS.15.2.17383

[9] Kim, M. (2019) Using Clinical Documentation Improvement to Improve Patient Care. Insights (Essays).

[10] Médecins Sans Frontières (2017) Atient Support, Education and Counselling Guideline for Adults Living with HIV and/or TB MSF January 2017. Contract No. 150917, Médecins Sans Frontières, Geneva.

[11] Gajera, G., Srinivasa, P., Ameen, S. and Gowda, M. (2019) Newer Documentary Practices as per Mental Healthcare Act 2017. Indian Journal of Psychiatry, 61, S686S692.

[12] Adebamowo, C.A., Casper, C., Bhatia, K., Mbulaiteye, S.M., Sasco, A.J., Phipps, W., et al. (2014) Challenges in the Detection, Prevention, and Treatment of HIV-Associated Malignancies in Low- and Middle-Income Countries in Africa. Journal of Acquired Immune Deficiency Syndromes, 67, S17-S26.

https://doi.org/10.1097/QAI.0000000000000255

[13] Wiedenmayer, K., Summer, R.S., Mackie, C.A., Gous, A.S., Everard, M., et al. (2006) Developing Pharmacy Practice: A Focus on Patient Care. World Health Organization, Geneva.

[14] Spicer, N., Aleshkina, J., Biesma, R., Brugha, R., Caceres, C., Chilundo, B., et al. (2010) National and Subnational HIV/AIDS Coordination: Are Global Health Initiatives Closing the Gap between Intent and Practice? Globalization and Health, 6, Article No. 3. https://doi.org/10.1186/1744-8603-6-3

[15] Meehan, S.-A., Sloot, R., Draper, H.R., Naidoo, P., Burger, R. and Beyers, N. (2018) Factors associated with linkage to HIV care and TB treatment at community-based HIV testing services in Cape Town, South Africa. PLOS ONE, 13, e0195208. https://doi.org/10.1371/journal.pone.0195208

[16] Chamy, R., Nurul, A. and Fajrilhuda, Y. (2020) Factors That Cause Compliance Filling Medical Records in Hospitals. International Journal of Community Medicine and Public Health, 7, 4180-4184. 
https://doi.org/10.18203/2394-6040.ijcmph20204393

[17] James, J.T. (2013) A New, Evidence-Based Estimate of Patient Harms Associated with Hospital Care. Journal of Patient Safety, 9, 122-128.

https://doi.org/10.1097/PTS.0b013e3182948a69

[18] Louden, D.T., Stuart, A.E. and Yemane, L. (2015) Improved Cycle Times in a Resident Continuity Clinic: A Resident Led Process Improvement Project. Academic Pediatrics, 15, E10-E11. https://doi.org/10.1016/j.acap.2015.04.031

[19] Pourasghar, F., Malekafzali, H., Koch, S. and Fors, U. (2008) Factors Influencing the Quality of Medical Documentation When a Paper-Based Medical Records System Is Replaced with an Electronic Medical Records System: An Iranian Case Study. International Journal of Technology Assessment in Health Care, 24, 445-451.

[20] Boissel, J., Nony, P., Amsallem, E., Mercier, C., Estève, J. and Cucherat, M. (2005) How to Measure Non-Consistency of Medical Practices with Available Evidence in Therapeutics: A Methodological Framework. Fundamental \& Clinical Pharmacolo$g y$, 19, 591-596. https://doi.org/10.1111/j.1472-8206.2005.00352.x

[21] World Health Organization (2006) Guidelines on Cotrimoxazole Prophylaxis for HIV-Related Infections among Children, Adolescents and Adults in Resource Limited Settings. World Health Organization, Geneva.

[22] Kamuhabwa, A. and Manyanga, V. (2015) Challenges Facing Effective Implementation of Co-Trimoxazole Prophylaxis in Children Born to HIV-Infected Mothers in the Public Health Facilities. Drug, Healthcare and Patient Safety, 7, 147-156.

https://doi.org/10.2147/DHPS.S89115

[23] Mutshatshi, T.E., Mothiba, T.M., Mamogobo, P.M. and Mbombi, M.O. (2018) Record-Keeping: Challenges Experienced by Nurses in Selected Public Hospitals. Curationis, 41, Article No. a1931. https://doi.org/10.4102/curationis.v41i1.1931 